\title{
Validity and reliability evidence of a point of care assessment of salivary cortisol and a-amylase: a pre-registered study
}

\author{
Kagan J Ducker ${ }^{1}$, Robin L.J. Lines ${ }^{1}$, Michael T Chapman ${ }^{1}$, Peter Peeling ${ }^{2}$, Alannah K.A. McKay ${ }^{2,3}$, Daniel F \\ Gucciardi ${ }^{\text {Corresp. } 1}$ \\ ${ }^{1}$ School of Physiotherapy and Exercise Science, Curtin University, Perth, Western Australia, Australia \\ 2 School of Human Sciences (Exercise and Sport Science), The University of Western Australia, Perth, Western Australia, Australia \\ 3 Australian Institute of Sport, Belconnen, Australian Capital Territory, Australia \\ Corresponding Author: Daniel F Gucciardi \\ Email address: daniel.f.gucciardi@gmail.com
}

Purpose: The iPro Cube is a small portable point-of-care device designed to analyse salivary markers of stress in a user-friendly manner (e.g., fast, convenient). Our aim was to test the reliability and validity of the iPro Cube LFD to measure salivary cortisol and aamylase as compared to the common laboratory standard method (ELISA immunoassay) prior to and after moderate intensity exercise. Methods: The study was a repeated measures, pre-registered design, and statistical framework that incorporated prior knowledge directly into the estimation process. Twenty-nine individuals (age $=27.4 \pm 6.6 \mathrm{y}$; body-mass $=70.8 \pm 11.3 \mathrm{~kg}$; height $=1.74 \pm 0.92 \mathrm{~m} ; 18$ males) completed a single $\mathrm{PWC}_{75 \% \mathrm{HRmax}}$, with repeated measures of salivary cortisol and a-amylase pre, immediately post, and 30 min post-exercise. Results: Correlation between the iPro and laboratorybased assessments of salivary cortisol was moderate-to-large $(0.53>r<0.81)$ across all three testing points. In contrast, correlation between the iPro and laboratory-based assessments of a-amylase was small-to-moderate $(0.25>r<0.46)$. We found a large correlation between duplicate samples of iPro cortisol assessment $(0.75>r<0.82)$, and a moderate-to-large correlation for a-amylase $(0.51>r<0.77)$. Conclusions: The iPro Cube LFD reader is capable of taking measures of salivary cortisol that are moderately correlated to values obtained via ELISA immunoassay, however the unit underestimates salivary cortisol and overestimates salivary a-amylase at rest and post-moderate intensity exercise. It is recommended that researchers continue using standard laboratory techniques to assess these salivary stress markers. 
1 Running head: iPro validity and reliability

2

3

4

5

6

$7 \quad$ Validity and reliability evidence of a point of care assessment of salivary cortisol and aamylase: a pre-registered study

a School of Physiotherapy and Exercise Science, Curtin University, Perth, Western Australia, Australia.

${ }^{\mathrm{b}}$ School of Human Sciences (Exercise and Sport Science), The University of Western Australia, Crawley, Western Australia, Australia. Corresponding author: Daniel Gucciardi, School of Physiotherapy and Exercise Science,

21 Curtin University, GPO Box U1987, Perth, Australia, 6845. Email:

22 daniel.f.gucciardi@gmail.com. Phone: +61 89266 3653. Supporting documentation (e.g., power 
23 simulations, data, analysis scripts) is available on the Open Science Framework:

24 http://bit.ly/2HCA6MR

25 


\section{Abstract}

27 Purpose: The iPro Cube is a small portable point-of-care device designed to analyse salivary markers of stress in a user-friendly manner (e.g., fast, convenient). Our aim was to test the reliability and validity of the iPro Cube to measure salivary cortisol and a-amylase as compared to the common laboratory standard method (ELISA immunoassay) prior to and after moderate intensity exercise.

32 Methods: The study was a repeated measures, pre-registered design, and statistical framework that incorporated prior knowledge directly into the estimation process. Twenty-nine individuals (age $=27.4 \pm 6.6 \mathrm{y}$; body-mass $=70.8 \pm 11.3 \mathrm{~kg}$; height $=1.74 \pm 0.92 \mathrm{~m} ; 18$ males $)$ completed a single $\mathrm{PWC}_{75 \% H R \max }$, with repeated measures of salivary cortisol and a-amylase pre, immediately post, and 30 min post-exercise.

Results: Correlation between the iPro Cube and laboratory-based assessments of salivary cortisol was moderate-to-large $(0.53>r<0.81)$ across all three testing points. In contrast, correlation between the iPro Cube and laboratory-based assessments of a-amylase was small-tomoderate $(0.25>r<0.46)$. We found a large correlation between duplicate samples of iPro Cube cortisol assessment $(0.75>r<0.82)$, and a moderate-to-large correlation for a-amylase $(0.51>r<0.77)$.

Conclusions: The iPro Cube is capable of taking measures of salivary cortisol that are moderately correlated to values obtained via ELISA immunoassay, however the unit underestimates salivary cortisol and overestimates salivary a-amylase at rest and post-moderate intensity exercise. It is recommended that researchers continue using standard laboratory

47 techniques to assess these salivary stress markers. 
50 Keywords: stress, psychophysiology, saliva, exercise 
51

52

\section{Introduction}

The stress placed on the body by exercise can be measured using various biological markers in saliva, hair, and blood. Two key systems in the neuroendocrine response to stress are the hypothalamic-pituitary-adrenal (HPA) axis and the autonomic nervous system (ANS), which drive responses to assist the body in coping with the applied stressor (Rutherford-Markwick, Starck, Dulson, \& Ali, 2017; Strahler, Skoluda, Kappert, \& Nater, 2017). Salivary markers of HPA axis and ANS activity have increased in popularity recently, as there are several metabolites that can be detected via this non-invasive technique, using only small sample volumes (i.e., $\sim 0.5-1 \mathrm{ml}$ ) (Lee, Kim, \& Choi, 2015; Papacosta \& Nassis, 2011). Collection of salivary metabolites offers a viable non-invasive alternative to serum and plasma methods where frequent venepuncture to sample blood may impact the analyte of interest (e.g., increasing cortisol response) or may be inappropriate, requiring less medical expertise, rapid collection, and can be performed in a variety of settings. Salivary cortisol (HPA axis) and alpha-amylase (aamylase; ANS) are two such markers that can be measured in the saliva as a surrogate measure of psychophysiological stress.

Cortisol is a glucocorticoid released from the adrenal cortex by activation of the HPA axis when the body is stressed (Lee et al., 2015). Only a thin layer of cells separate the salivary ducts from blood passing through the area, which ultimately results in trace levels of cortisol passing through the oral cavity into saliva; when measured, salivary cortisol typically provides a strong representation of plasma cortisol levels (Törnhage, 2009), though disassociations have been observed under challenge situations, during the circadian cycle, and in terms of free versus protein-bound cortisol concentrations (Levine, Zagoory-Sharon, Feldman, Lewis, \& Weller, 2007). Salivary cortisol is advocated for use within stress research because of the generally 
74

strong representation of plasma cortisol levels and stress free nature of collection (Hellhammer, Wüst, \& Kudielka, 2009). Cortisol has widespread effects (e.g., stimulation of protein and lipid catabolism, increased gluconeogenesis and reduced inflammation) that are aimed at enhancing the ability of the body to cope with stress and repair tissue damage (Lee et al., 2015; Levine et al., 2007). Importantly, when the HPA axis is chronically stimulated, the typical responsiveness of the axis may be supressed, which may inhibit immune function (Adam et al., 2017). The responsiveness of the HPA axis makes cortisol a useful marker for assessing the acute (shortterm) and chronic (long-term) response to stress in individuals and athletes.

Alternatively, a-amylase is an enzyme that is secreted directly into the saliva in response to ANS activation, as would commonly occur when humans experience psychophysiological stress (Bosch, Veerman, de Geus, \& Proctor, 2011; Nater \& Rohleder, 2009). Importantly, the salivary concentration has a consistent circadian rhythm and is highly responsive to changes in ANS activity as it is produced within the salivary glands rather than being transported through into the salivary ducts from plasma like cortisol (Nater \& Rohleder, 2009; Rohleder \& Nater, 2009). During exercise, and particularly during high-intensity efforts, the ANS is activated, thereby making a-amylase a potentially useful indicator of physiological stress in humans. Caution is advised when using a-amylase as a measure of ANS activity (Bosch et al., 2011), as stress induced increases in a-amylase have been found to correlate poorly with other indicators of ANS activity (e.g., catecholamines)(Thoma, Kirschbaum, Wolf, \& Rohleder, 2012).

Nevertheless, this poor degree of association is a common issue with indicators of ANS activity, and in turn is not considered a disqualifying factor (Engeland, Bosch, \& Rohleder, 2019).

Typically, stress markers such as cortisol and a-amylase are assessed via a sample of saliva or blood, which are analysed in a laboratory by highly skilled technicians using processes 
97 that are expensive and can take several hours or days. However, small portable point-of-care

98 (POC) devices are available to make this process easier to conduct in the field,

with analysis requiring short timeframes that allow the rapid generation of information to quickly assess the stress response. Recently, Soma Bioscience (Oxfordshire, UK) have released two lateral flow device (LFD) readers, the iPro Lab ${ }^{1}$ and iPro Cube ${ }^{2}$. Immunochromagraphic strips (lateral flow devices $-\mathrm{LFD}^{3}$ ) containing a test line that responds to gold labelled antisecretory antibodies are exposed to a saliva sample (and buffer) which can be assessed by the reader to obtain a measure of salivary cortisol and a-amylase. The iPro Lab has been shown to be

iPro Lab's utility in measuring salivary cortisol has been validated against traditional methods,

( $p=0.881)$ and the passive drool method $(p=0.145)$ (Fisher et al., 2015). Evidence also

${ }^{1}$ iPro Lab reader - this is iPro's larger, and older, saliva analysis system. It reads the lateral flow device (LFD) that contains the samples of saliva. See http://somabioscience.com/?page id=214. We will simply refer to this as the iPro Lab.

2 iPro Cube reader - this is iPro's smaller, and newer, portable saliva analysis system. It reads the lateral flow device (LFD) that contain the samples of saliva. See http://somabioscience.com/?page id=182. We will simply refer to this as the iPro Cube.

${ }^{3}$ iPro Cube LFD - We will simply refer to this as a lateral flow device (LFD). The LFD's are the strips that contain the saliva samples and are inserted into either the iPro Lab or Cube devices to analyse the saliva samples. 
116 (intermittent) exercise (Coad et al., 2016; Coad, Gray, et al., 2015). However, whether the newer

117 and more portable version of this device (iPro Cube) can be used to assess a-amylase at rest, and

118 to measure relatively small changes in salivary cortisol and a-amylase post-moderate intensity

119 exercise remains unknown. This information is important for researchers, sports scientists, and

120 health professionals who are seeking a POC device to assess markers of psychophysiological

121 stress in a field setting. Therefore, our aim was to test in the field the reliability and validity of

122 the iPro Cube to measure salivary cortisol and a-amylase as compared to the common laboratory

123 standard method (ELISA). On the basis of a conservative estimate from past research (Coad,

124 McLellan et al., 2015; Fisher et al., 2015), we hypothesised that;

125 1. For salivary cortisol and a-amylase, respectively, POC assessment will correlate positively

126 and moderately ( $r \sim 0.50)$ with a laboratory-based assessment (a) pre-exercise (resting

127 baseline), (b) immediately post-exercise, and (c) $30 \mathrm{~min}$ post-exercise.

128 2. Duplicate samples collected concurrently of the POC assessment of salivary cortisol and a-amylase, respectively, will correlate positively and strongly $(r \sim 0.80)$.

Materials and Methods

131

132

133

134

135

136

137

138

\section{Participants}

Monte Carlo simulations (L. K. Muthén \& Muthén, 2002) indicated that 15 participants would provide sufficient power $(100 \%)$ to detect a conservative effect size $(r \sim 0.50)$ when incorporating prior beliefs informed by past research (Coad, McLellan, et al., 2015; Fisher et al., 2015) directly into the estimation process. Full details of these power simulations are available on the Open Science Framework (OSF; http://bit.ly/2HCA6MR). Individuals were eligible to participate if they were university students aged $\geq 18$ years; we excluded people from taking part in the study if they had an illness or injury that prevented them from completing a sub-maximal 
139 exercise test. In total, 29 eligible individuals provided written informed consent and took part in

140 this study (age $=27.4 \pm 6.6 \mathrm{y}$, range $=19-48 \mathrm{y}$; body-mass $=70.8 \pm 11.3 \mathrm{~kg}$; height $=1.74 \pm 0.92 \mathrm{~m}$;

$141 \mathrm{BMI}=23 \pm 3 ; 18$ males). The study was approved by Curtin University's Human Ethics Research

142 Committee (HRE2017-0518).

\section{Procedures}

All study hypotheses, methods, procedures and data analysis plans were pre-registered on 145 the OSF (https://osf.io/tshkc/). The study design was a single-session exercise protocol with 146 repeated measures of salivary cortisol and a-amylase prior to completing a sub-maximal test of

147 aerobic work capacity, immediately following this test, and after $30 \mathrm{~min}$ of rest. Each participant 148 attended one testing session (08:00 - 10:00 or 10:00 - 12:00) to minimise any effect from 149 circadian variations. Waking time was not recorded, however, there should have been $>60 \mathrm{~min}$ 150 between the participants waking to the start of salivary sampling, to ensure that the cortisol 151 awakening response was avoided. Upon arriving in the laboratory and providing informed 152 consent, participants completed a short survey including self-reported demographic information 153 (e.g., gender and age). Participants next completed a sub-maximal test of aerobic work capacity 154 (PWC $_{75 \% H R m a x}$; Gore et al. 1999; Miyashita et al. 1985) where there would be some stimulation of the HPA. Briefly, this test involves completing three workloads of 3-6 min at approximately 55,65 and $75 \%$ of age predicted maximum heart rate $(220 \mathrm{bpm}$ - age in years) on a cycle ergometer (Monark, Monark Exercise AB, Varnsbro, Sweden), which was used as a method of prescribing a bout of individualised moderate intensity physical activity. This validation study was part of a larger project our team was conducting with Army personnel, so we decided to focus on an intensity that was most representative of typical training scenarios (Friedl et al., 161 2012). Previous studies have reported increases in both salivary cortisol and a-amylase in 
162 response to a single bout of exercise (Strahler et al., 2017). Heart rate and ratings of perceived 163 exertion (Borg, 1982) were collected each minute during the test.

Participants were required to avoid high-intensity exercise and alcohol for $24 \mathrm{~h}$ and

165

166

167

168

169

170

171

172

173

174

175

176

177

178

179

180

181

182

183

184

brushing their teeth or eating food for $2 \mathrm{~h}$ pre-exercise. Samples of saliva were taken pre-warmup, immediately post-exercise and 30 min post-exercise. Participants rinsed their mouth with water 10 min prior to saliva collection at pre-warm-up. Nine unstimulated whole saliva samples were provided by each participant $(3 \times$ pre-warm-up, $3 \times$ immediately post-exercise and $3 \times 30$ min post-exercise). On each occasion duplicate oral swabs (iPro oral fluid collector; Soma Bioscience, Oxfordshire, UK) were placed in the mouth concurrently for assessment using a cortisol/a-amylase LFD before being analysed using a LFD reader (iPro Cube, Soma Bioscience, Oxfordshire, UK). The intra-sample coefficient of variation (CV) for the iPro salivary cortisol aamylase samples were 8.8 and $20.0 \%$, respectively. Next, the third sample was collected by the passive drool method directly into a $2 \mathrm{~mL}$ cryovial immediately after the sample for the iPro Cube (Salimetrics LLC, Pennsylvania, USA). This sample was frozen at $-20^{\circ} \mathrm{C}$ until analysis in duplicate for cortisol and a-amylase using a validated, commercially available enzyme-linked immunosorbent assay (ELISA; Salimetrics, USA) by Stratech Scientific APAC Pty Ltd. (Sydney, Australia). Freezing has minimal influence of samples at $-20^{\circ} \mathrm{C}$ even after one year in storage (Garde \& Hansen, 2005). The inter-assay and intra-assay CV for the salivary cortisol samples was 5.0 and 4.4\%, respectively. The inter-assay and intra-assay CV for the salivary aamylase samples was 5.8 and $5.1 \%$, respectively.

\section{Statistical Analyses}

The registered study hypotheses were tested in Mplus 8 (L. K. Muthén \& Muthén, 19982017) using a series of bivariate correlations with a Bayesian estimator. The execution and 
185 reporting of these analyses were informed by recent guidelines for Bayesian statistics (Depaoli \&

186 van de Schoot, 2017). An overview of the priors for each hypothesis is available on the OSF. We

187 also report and compare our weakly or informative priors with non-informative (diffuse) priors

188 to examine the effect of prior information on the posterior distribution (Depaoli \& van de Schoot, 189 2017). Briefly, the specification of non-informative priors allows the data at hand to drive the

190 estimations within the posterior distribution because it reflects substantial uncertainty regarding

191 the nature of the target parameter. Model convergence was assessed using statistical (i.e.,

192 potential scale reduction factor; PSR <1.05) and visual criteria (i.e., inspection of trace plots for

193 stability in mean and variance of each chain). Posterior predictive checking is used to assess

194 model fit in Bayesian estimation, where the posterior distribution is compared with the observed

195 data to examine the degree to which the replicated data matches the observed data (B. O. Muthén

196 \& Asparouhov, 2012). The posterior predictive $p$-value (PPP) and associated 95\% credibility

197 interval (CI) is produced in Mplus; values close to 0.50 reflect an excellent fitting model, though

198 typically values greater than 0.05 are considered acceptable (B. O. Muthén \& Asparouhov,

199 2012). Parameter estimates are considered credible when the 95\% CI excludes zero. We

200 classified "not a detectable" (NAN) readings as a missing value; missing data were handled with

201 the Gibbs sampler that treats the missing observations as unknown values to be estimated and the

202 algorithm used will correctly estimate the model under the missing at random (MAR)

203 assumption (Asparouhov \& Muthén, 2010). All Mplus output files including analysis syntax

204 together with Bland-Altman plots (Bland \& Altman, 1986) are available on the OSF

205 (https://osf.io/tshkc/).

206

\section{Results}


The raw data file is available on the OSF. Descriptive statistics for the heart rate and

208

perceived exertion response to the exercise stimulus are presented in Table 1. In total, 49 (47 of which were obtained with the iPro Cube) of 522 data points were recoded as missing due to an undetectable reading; 24 of the iPro Cube samples were recoded as 0 when the device provide a value that was $<0.7$.

Group-level descriptive statistics for salivary cortisol and a-amylase are presented in Table 2. Analysis of the Bland-Altman plots (available on the OSF; https://osf.io/tshkc/) show a positive mean difference in cortisol concentrations at all time points of the assessment, indicating that the iPro Cube underestimates salivary cortisol. Additionally, the difference between the iPro Cube and immunoassay increases with greater cortisol concentrations. A negative mean difference in $\alpha$-amylase was also observed, indicating that the iPro Cube overestimates $\alpha$ amaylase. Further, this difference becomes greater with increasing levels of $\alpha$-amaylase.

With regard to the main analyses, the probability of the data, given the hypothesised model, was acceptable for all models associated with each hypothesis (i.e., PPP >0.20). Inspections of the trace plots and PSR evolution through the simulations (i.e., started within 0.50 of 1 and reduced quickly close to 1 and remained stable throughout the entire sequence of iterations) verified support for model convergence. Full details of model-data fit and convergence are available in the output and supplementary material files located on the OSF. Parameter estimates of all models tested using weakly informative (uniform), informative, and non-informative (diffuse) priors are displayed in Table 3. We also report in Table 3 parameter estimates obtained using a robust maximum likelihood estimator for readers most familiar with frequentist statistics. 
POC and laboratory-based assessments of salivary cortisol was moderate-to-large $(0.53>r<0.81)$

231

232

233

234

235

236

237

238

239

240

241

242

243

244

245

246

247

248

249

250

251

across all three testing points. In contrast, correlation between POC and laboratory-based

assessments of a-amylase was small-to-moderate in magnitude $(0.25>r<0.46)$. A similar trend in the findings was observed for hypothesis 2 , where we found large correlation between duplicate samples of POC assessment of cortisol $(0.75>r<0.82)$, but moderate-to-large correlation aamylase $(0.51>r<0.77)$. A closer inspection of the findings revealed variation in the substantive interpretation of the results in two ways. First, the strength of association between the POC assessment and laboratory analysis of salivary cortisol and a-amylase varied across the duplicate samples at each assessment point (24-50\%). Second, the discrepancies between the weakly informative and informative priors with the non-informative prior indicated moderate-to-large effects of the prior information on the posterior distribution, which represents a mismatch between prior expectation and the data at hand (Depaoli \& van de Schoot, 2017).

\section{$\sqrt{10}$}

We tested the reliability and validity of the iPro Cube as a rapid field assessment of salivary cortisol and a-amylase, comparing the outcomes to the most common laboratory method (ELISA) utilising a pre-registered design and statistical framework that incorporated prior knowledge directly into the estimation process. Our findings revealed mixed support for the hypotheses.

During the exercise testing, participants experienced peak heart rates reflective of moderate- to vigorous-intensity exercise (Norton, Norton, \& Sadgrove, 2010), and RPE that were indicative of light to somewhat hard efforts (Borg, 1982) (see Table 1). Considering that this test was graded, and only involved short workloads, the overall physical stress placed on the 
252 participants was moderate, but only for a short period. Although the response of salivary cortisol

253 to exercise can be variable, previous research suggests that moderate- to vigorous-intensity

254 exercise, particularly when prolonged in nature, will stimulate the HPA and cause an increase in

255 salivary cortisol (Hayes, Grace, Baker, \& Sculthorpe, 2015). However, due to the short duration

256 and low to moderate initial workloads of the $\mathrm{PWC}_{75 \% \mathrm{HRmax}}$, in our participants, a slight trend for

257 a decrease in salivary cortisol may indicate that the stimulus was not stressful enough to activate

258 the HPA axis. If the HPA axis was insufficiently stimulated to achieve an increase, the slight

259 decrease may instead be reflective of the typical diurnal variation, particularly in the 10:00

260 participants, as cortisol decreases rapidly after an initial increase post-awakening (Clow, Thorn,

261 Evans, \& Hucklebridge, 2004). Both Van Brugger et al. (2011) and Jacks et al. (2002) identified

262 no increase in salivary cortisol during low $\left(40-45 \% \dot{\mathrm{VO}}_{2 \text { peak }}\right)$ or moderate-intensity $(60 \% \dot{\mathrm{V}}$

$\left.263 \mathrm{O}_{2 \text { peak }}\right)$ cycling for 30-60 min, but did see increases during high-intensity $(75-80 \% \dot{\mathrm{VO}} 2$ peak $)$

264 cycling. The intense and prolonged (in the case of Jacks et al. 2002) nature of the high-intensity

265 stimulus in these studies is likely responsibly for the increases in salivary cortisol observed.

266 The utility of the newer iPro Cube for assessing salivary cortisol in the field has yet to be

267 assessed. In the current study, moderate to large correlations were found between the salivary

268 cortisol measures obtained from the iPro Cube and immunoassay. Additionally, a large

269 correlation was identified between duplicate saliva samples analysed using the iPro Cube.

270 However, although the measures may be related, and duplicate samples are similar for the iPro

271 Cube, there was a mean difference of 51.5-64.6\% (underestimate) between the iPro Cube and

272 the immunoassay results. As noted in the data file available on the OSF, 47 of 348 possible

273 samples returned an undetectable result from the iPro Cube. Importantly, the immunoassay has a

274 lower reported limit of quantitation (LOQ) for salivary cortisol (ELISA immunoassay $=0.19$ 
$275 \mathrm{nmol} \cdot \mathrm{L}^{-1}$ vs. iPro Cube $=0.58 \mathrm{nmol} \cdot \mathrm{L}^{-1}$; Salimetrics, 2019, Soma Bioscience, 2019). These

276 findings raise concerns regarding the reliability and validity of the iPro Cube to assess salivary

277 cortisol and a-amylase. Fisher et al. (2015) have previously reported that the iPro Lab and OFC

278 swab collection method provides valid results compared to ELISA when using both passive

279 drool $(r=0.45)$ and Salimetrics oral swab $(r=0.52)$ to collect the sample. Additionally, they

280 identified that the OFC swab and Lab LFD were reliable when assessing duplicate samples (OFC

281 swab duplicates, $\mathrm{p}=0.81, \mathrm{ICC}=0.89$; iPro Lab duplicates, $\mathrm{p}=0.98, \mathrm{ICC}=0.85$ ). However, it is

282 important to bear in the mind the sensitivity of their design (small sample) and relatively weak

283 correlations between methods. The reason for the difference in our findings is unclear as the

284 measurement and analysis techniques were similar, however, the contrast may be related to

285 issues with the manufacturer supplied buffer solution, LFD's, or differences between the

286 performance of the two models of iPro LFD readers.

287 Alpha-amylase concentrations were found to increase post-exercise before returning to

288 baseline 30 min post-exercise in the current study. Previous research has suggested that a-

289 amylase is a sensitive marker of ANS activation as it is produced locally in the salivary glands

290 rather than being a systemic marker (Rohleder \& Nater, 2009). Our results support the literature

291 in suggesting that a-amylase is sensitive to changes in stress induced by exercise, even during

292 short-duration exercise efforts at moderate intensity. For example, Allgrove et al. (2008) reported

293 that salivary a-amylase concentrations were increased while cycling at an intensity as low as

$29450 \% \dot{\mathrm{VO}_{2 \max }}$ for $\sim 22 \mathrm{~min}$, and were undifferentiated from concentrations recorded at $75 \% \dot{\mathrm{V}}$

$295 \mathrm{O}_{2 \max }$, or exercise to fatigue during a graded exercise test. Similarly, Kunz et al. (2015) identified

296 that a-amylase was increased during a cycle for $30 \mathrm{~min}$ at $-5,+5$ and $+15 \%$ of the blood lactate

297 threshold in experienced cyclists, yet the change in concentration was significantly greater in the 
$298+15 \%$ condition when compared to the $-5 \%$ condition even though concentrations were similar

299 between trials. With this in mind, future research should investigate the sensitivity of a-amylase

300 to detect changes in exercise intensity, particularly during short duration and low to moderate-

301 intensity efforts.

302 This study is the first to report the efficacy of using either of the iPro LFD readers to 303 assess a-amylase at rest and following moderate-intensity exercise. Importantly, it was identified 304 that the validity of the iPro Cube was poor as it was identified that values obtained did not 305 correlate well with the immunoassay $(0.50>\mathrm{r}<0.77)$ or between repeated concurrent samples $306(0.25>r<0.46)$. Furthermore, the $\mathrm{CV}$ within samples was very high for the POC device. Although 307 there are differences in the way that each method determines a-amylase, converting both sets of 308 results to enzyme activity using the manufacturer's guidelines should lead to comparable results.

309 The POC unit over-estimated a-amylase activity by approximately $150 \%$, which generates

310 concern about the analysis process and/or LFD strips and reader. Early testing for a-amylase 311 with the unit encountered consistent "not a number - NAN" error messages, which may relate to

312 the unit measuring what it thought were very high or low concentrations of a-amylase. In this 313 instance, the manufacturer checked the buffering solutions and calibration (unique to each unit 314 and buffer solution), which reduced the NAN error messages in subsequent trials, but evidently 315 failed to allow an accurate or consistent assessment of a-amylase by the unit. Importantly, the 316 LOQ for the immunoassay is reported $\left(0.58 \mathrm{nKat} \cdot \mathrm{L}^{-1}\right.$; Salimetrics, 2019), yet we were unable to 317 locate this value for the iPro Cube.

318 This study had several limitations that should be considered. First, we did not assess the 319 waking time of our participants; although we presume that participants in the first test group 320 (08:00) should have surpassed the peak of the cortisol awakening response, we cannot guarantee 
321 that this didn't impact our initial resting measurement in this group. Second, differences in the

322 collection method may explain some variability in the concentrations of cortisol and $\alpha$-amylase.

323 Although the manufacturer has reported that their oral fluid collector recovers $>85 \%$ of analytes

324 (IgA, cortisol, testosterone and DHEA) in a sample versus passive drool (Jehanli, Dunbar, \&

325 Skelhorn, 2011), this finding is yet to be supported in published, independent research,

326 particularly with $\alpha$-amylase. The assessment of salivary markers is commonly completed using

327 ELISA immunoassay, and immunoassay could potentially be considered the laboratory standard

328 for these assessments, yet liquid-chromatography mass spectroscopy (LC-MSMS) is typically

329 considered the gold standard (Inder, Dimeski, \& Russell, 2012). It is likely these techniques

330 would produce similar results, yet future research should look to compare the measures obtained

331 using the iPro, ELISA and LC-MSMS. Additionally, comparisons between the more commonly

332 used iPro Lab and new iPro Cube would be beneficial to determine if the units return similar 333 results.

334

335

336

337

338

339

340

341

342 343

\section{Conclusion}

We identified that the iPro Cube reader is capable of reporting measures of salivary cortisol that are moderately correlated to values obtained via the typical laboratory method (ELISA immunoassay). However, the unit was shown to underestimate salivary cortisol and overestimate salivary a-amylase at rest and post-moderate intensity exercise when compared to the common laboratory standard ELISA approach. Collectively, therefore, the current findings provide little support for the utility of the iPro Cube as a tool for measuring salivary cortisol and a-amylase in the field. As such, researchers, sports scientists, and health professionals are recommended to continue using standard laboratory techniques to assess these salivary stress markers.

Peer] reviewing PDF | (2019:08:40185:2:0:NEW 28 Nov 2019) 
Adam, E. K., Quinn, M. E., Tavernier, R., McQuillan, M. T., Dahlke, K. A., \& Gilbert, K. E. (2017). Diurnal cortisol slopes and mental and physical health outcomes: a systematic review and meta-analysis. Psychoneuroendocrinology, 83, 25-41. doi:10.1016/j.psyneuen.2017.05.018

Allgrove, J. E., Gomes, E., Hough, J., \& Gleeson, M. (2008). Effects of exercise intensity on salivary antimicrobial proteins and markers of stress in active men. Journal of Sports Sciences, 26(6), 653-661. doi:10.1080/02640410701716790

Asparouhov, T., \& Muthén, B. (2010). Bayesian analysis using Mplus: Technical implementation. Retrieved from http://www.statmodel.com/download/Bayes3.pdf

Bland, J. M., \& Altman, D. G. (1986). Statistical methods for assessing agreement between two methods of clinical measurement. Lancet, 1, 307-310.

Borg, G. (1982). Psychophysical bases of perceived exertion. Medicine and Science in Sports and Exercise, 14, 377-381. doi:10.1249/00005768-198205000-00012

Bosch, J. A., Veerman, E. C., de Geus, E. J., \& Proctor, G. B. (2011). $\alpha$-Amylase as a reliable and convenient measure of sympathetic activity: don't start salivating just yet! Psychoneuroendocrinology, 36, 449-453.

Clow, A., Thorn, L., Evans, P., \& Hucklebridge, F. (2004). The awakening cortisol response:

Coad, S., Gray, B., \& McLellan, C. (2016). Seasonal analysis of mucosal immunological 366 function and physical demands in professional Australian Rules footballers. International Journal of Sports Physiology and Performance, 11, 574-580. doi:10.1123/ijspp.2015-0242 
368 Coad, S., Gray, B., Wehbe, G., \& McLellan, C. (2015). Physical demands and salivary

369 immunoglobulin A responses of elite Australian Rules football athletes to match play.

370 International Journal of Sports Physiology and Performance, 10, 613-617.

371 doi:10.1123/ijspp.2014-0493

372 Coad, S., McLellan, C., Whitehouse, T., \& Gray, B. (2015). Validity and reliability of a novel 373 salivary immunoassay for individual profiling in applied sports science. Research in Sports 374 Medicine, 23(2), 140-150. doi:10.1080/15438627.2015.1005300

375 Depaoli, S., \& van de Schoot, R. (2017). Improving transparency and replication in Bayesian 376 statistics: The WAMBS-checklist. Psychological Methods, 22, 240-261.

377 doi: $10.1037 /$ met0000065

378 Engeland, C. G., Bosch, J. A., \& Rohleder, N. (2019). Salivary biomarkers in 379 psychoneuroimmunology. Current Opinion in Behavioral Sciences, 28, 58-65.

380 Fisher, R. N., McLellan, C., \& Sinclair, W. H. (2015). The validity and reliability for a salivary 381 cortisol point of care test. Journal of Athletic Enhancement, 4(4). doi:10.4172/23243829080.1000204

383 Friedl, K. E., Knapik, J. J., Hakkinen, K., Baumgartner, N., Groeller, H., Taylor, N. A. S., . . . 384 Nindl, B. C. (2012). Perspectives on aerobic and strength influences on military physical 385 readiness: report of an international military physiology roundtable. Journal of Strength and $386 \quad$ Conditioning Research, 29, S10-S23.

387 Garde, A. H., \& Hansen, Å. M. (2005). Long-term stability of salivary cortisol. Scandinavian 388 Journal of Clinical and Laboratory Investigation, 65, 433-436. 
389 Gore, C. J., Booth, M. L., Bauman, A., \& Neville, O. (1999). Utility of pwc75\% as an estimate

390 of aerobic power in epidemiological and population-based studies. Medicine and Science in

391 Sports and Exercise, 31, 348-351. doi:10.1097/00005768-199902000-00020

392 Hayes, L. D., Grace, F. M., Baker, J. S., \& Sculthorpe, N. (2015). Exercise-induced responses in 393 salivary testosterone, cortisol, and their rations in men: a meta-analysis. Sports Medicine, 45, 394 713-726. doi:10.1007/s40279-015-0306-y

395 Hellhammer, D. H., Wüst, S., \& Kudielka, B. M. (2009). Salivary cortisol as a biomarker in 396 stress research. Psychoneuroendocrinology, 34, 163-171.

397 Inder, W. J., Dimeski, G., \& Russell, A. (2012). Measurement of salivary cortisol in 2012 -

398 laboratory techniques and clinical indications. Clinical Endocrinology, 77, 645-651.

399 doi:10.1111/j.1365-2265.2012.04508.x

400 Jacks, D. E., Sowash, J., Anning, J., McGloughlin, T., \& Andres, F. (2002). Effect of exercise at 401 three exercise intensities on salivary cortisol. Journal of Strength and Conditioning Research, $402 \quad 16(2), 286-289$.

403 Jehanli, A., Dunbar, J., \& Skelhorn, S. (2011). Development and validation of an oral fluid 404 collection device and its use in the immunoassay of salivary steroids and immunoglobins in 405 sports persons. Paper presented at the The International Society of Exercise Immunology

406 Symposium, Oxford, United Kingdom. http://www.isei.dk/index.php?pageid=21

407 Kunz, H., Bishop, N. C., Spielmann, G., Pistillo, M., Reed, J., Ograjsek, T., . . Simpson, R. J.

408 (2015). Fitness level impacts salivary antimicrobial protein responses to a single bout of 409 cycling exercise. European Journal of Applied Physiology, 115, 1015-1027.

410 doi:10.1007/s00421-014-3082-8 
411 Lee, D. Y., Kim, E., \& Choi, M. H. (2015). Technical and clinical aspects of cortisol as a 412 biochemical marker of chronic stress. Biochemistry and Molecular Biology Reports, 48(4), 209-216. doi:10.5483/BMBRep.2015.48.4.275

414 Levine, A., Zagoory-Sharon, O., Feldman, R., Lewis, J. G., \& Weller, A. (2007). Measuring 415 cortisol in human psychobiology studies. Physiology and Behaviour, 90(1), 43-53.

416 doi:10.1016/j.physbeh.2006.08.025

417 MacDonald, L. A., Bellinger, P. M., \& Minahan, C. L. (2017). Reliability of salivary cortisol and 418 immunoglobulin-A measurements from the IPRO ${ }^{\circledR}$ before and after sprint cycling exercise. 419 Journal of Sports Medicine and Physical Fitness, 57(12), 1680-1686. doi:10.23736/S0022$420 \quad 4707.16 .06785-2$

421 Miyashita, M., Mutoh, Y., Yoshioka, N., \& Sadamoto, T. (1985). PWC75\%HRmax: a measure 422 of aerobic work capacity. Sports Medicine, 2, 159-164. doi:10.2165/00007256-198502030$423 \quad 00001$

424 Muthén, B. O., \& Asparouhov, T. (2012). Bayesian structural equation modelling: A more 425 flexible representation of substantive theory. Psychological Methods, 17, 313-335. 426 doi:10.1037/a0026802

427 Muthén, L. K., \& Muthén, B. (1998-2017). Mplus users guide (8th ed.). Los Angeles CA: $428 \quad$ Muthén \& Muthén.

429 Muthén, L. K., \& Muthén, B. O. (2002). How to use a Monte Carlo study to decide on sample $430 \quad$ size and determine power. Structural Equation Modelling, 9(4), 599-620. doi:10.1207/S15328007SEM0904_8 
432 Nater, U. M., \& Rohleder, N. (2009). Salivary alpha-amylase as a non-invasive biomarker for the

433 sympathetic nervous system: current state of research. Psychoneuroendocrinology, 34, 486-

434 496. doi:10.1016/j.psyneuen.2009.01.014

435 Norton, K., Norton, L., \& Sadgrove, D. (2010). Position statement on physical activity and

436 exercise intensity terminology. Journal of Science and Medicine in Sport, 13, 496-502.

437 doi:10.1016/j.jsams.2009.09.008

438 Papacosta, E., \& Nassis, G. P. (2011). Saliva as a tool for monitoring steroid, peptide and

439 immune markers in sport and exercise science. Journal of Science and Medicine in Sport, 14,

$440 \quad$ 424-434. doi:10.1016/j.jsams.2011.03.004

441 Rohleder, N., \& Nater, U. M. (2009). Determinants of salivary a-amylase in humans and

442 methodological considerations. Psychoneuroendocrinology, 34, 469-485.

443 doi:10.1016/j.psyneuen.2008.12.004

444 Rutherford-Markwick, K., Starck, C., Dulson, D. K., \& Ali, A. (2017). Salivary diagnostic

445 markers in males and females during rest and exercise. Journal of the International Society of

446 Sports Nutrition, 14(1), 27-34. doi:10.1186/s12970-017-0185-8

447 Salimetrics. (2019a). Expanded Range High Sensitivity Salivary Cortisol Enzyme Immunoassay

448 Kit. Retrieved from https://salimetrics.com/wp-content/uploads/2018/03/salivary-cortisol-

$449 \quad$ elisa-kit.pdf

450 Salimetrics. (2019b). Salivary $\alpha$-Amylase Kinetic Enzyme Assay Kit. Retrieved from

451 https://salimetrics.com/wp-content/uploads/2018/03/alpha-amylase-saliva-elisa-kit.pdf

452 The Soma IgA/Cortisol Test With the SOMA Cube Reader. Retrieved from

$453 \quad$ http://somabioscience.com/?page $\mathrm{id}=281$ 
454 Strahler, J., Skoluda, N., Kappert, M. B., \& Nater, U. M. (2017). Simultaneous measurement of 455 salivary cortisol and alpha-amylase: application and recommendations. Neuroscience \& 456 Biobehavioral Reviews, 83, 657-677.

457 Thoma, M. V., Kirschbaum, C., Wolf, J. M., \& Rohleder, N. (2012). Acute stress responses in 458 salivary alpha-amylase predict increases of plasma norepinephrine. Biological psychology, 91, $459 \quad 342-348$.

460 Törnhage, C. J. (2009). Salivary cortisol for assessment of hypothalamic-pituitary-adrenal axis 461 function. Neuroimmunomodulation, 16, 284-289. doi:1021-7401/09/0165-0284\$26.00/0

462 VanBruggen, M. D., Hackney, A. C., McMurray, R. G., \& Ondrak, K. S. (2011). The 463 relationship between serum and salivary cortisol levels in reponse to different intensities of 464 exercise. International Journal of Sports Physiology and Performance, 6(3), 396-407. 465 doi:10.1123/ijspp.6.3.396 


\section{Table $\mathbf{1}$ (on next page)}

Descriptive statistics for heart rate, percentage of age predicted heart rate maximum, and rating of perceived exertion during workloads 1 - 3 of the $P W C_{75 \% H R m a x}$ test 
1 Table 1. Descriptive statistics for heart rate, percentage of age predicted heart rate maximum $\left(\% H R_{\max }=\right.$

2220 bpm-age in years), and rating of perceived exertion (RPE 6-20; Borg, 1982) during workloads 1 -

33 of the $P W C_{75 \% \text { HRmax }}$ test.

\begin{tabular}{|c|c|c|c|c|c|}
\hline & & \multicolumn{2}{|c|}{ Range } & \multirow[b]{2}{*}{ Mean } & \multirow[b]{2}{*}{$\mathrm{SD}$} \\
\hline & & Minimum & Maximum & & \\
\hline \multirow{3}{*}{$\begin{array}{l}\text { Heart rate } \\
\text { (bpm) }\end{array}$} & Workload 1 & 86 & 128 & 110 & 8 \\
\hline & Workload 2 & 97 & 158 & 129 & 11 \\
\hline & Workload 3 & 124 & 170 & 149 & 8 \\
\hline \multirow{3}{*}{$\% \mathrm{HR}_{\max }$} & Workload 1 & $50 \%$ & $64 \%$ & $56 \%$ & $3 \%$ \\
\hline & Workload 2 & $53 \%$ & $81 \%$ & $66 \%$ & $5 \%$ \\
\hline & Workload 3 & $70 \%$ & $87 \%$ & $77 \%$ & $4 \%$ \\
\hline \multirow{3}{*}{ RPE } & Workload 1 & 6 & 14 & 10 & 2 \\
\hline & Workload 2 & 6 & 17 & 12 & 3 \\
\hline & Workload 3 & 7 & 19 & 14 & 3 \\
\hline
\end{tabular}

4

$5 \quad$ Note: $\mathrm{bpm}=$ beats per minute; $\% \mathrm{HR}_{\max }=$ Percentage of maximum heart rate; $\mathrm{REP}=$ Rate of perceived

6 exertion.

7 


\section{Table 2 (on next page)}

Descriptive statistics for salivary cortisol and a-amylase pre-warm-up, immediately postexercise and 30 min post-exercise 
1 Table 2. Descriptive statistics for salivary cortisol and a-amylase pre-warm-up, immediately post-

2 exercise and $30 \mathrm{~min}$ post-exercise.

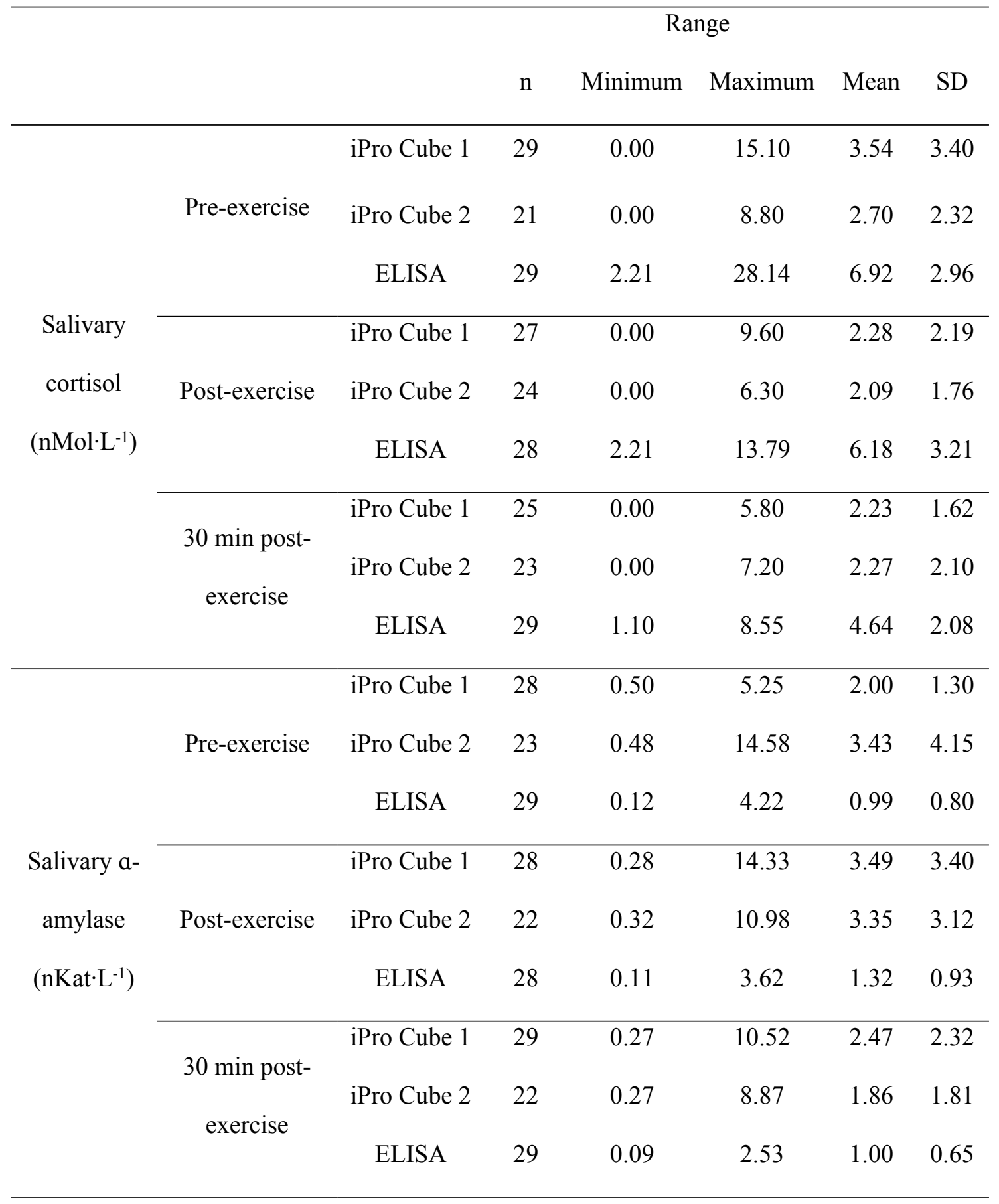

3 


\section{Table 3 (on next page)}

Comparison of standardised correlation coefficients between Bayesian and maximum likelihood estimators 
1 Table 3. Comparison of standardised correlation coefficients between Bayesian and maximum likelihood estimators.

\begin{tabular}{|c|c|c|c|c|c|c|c|c|c|}
\hline & & \multicolumn{2}{|c|}{$\begin{array}{l}\text { Bayesian Uniform/Weakly } \\
\text { Informative Prior }\end{array}$} & \multicolumn{2}{|c|}{ Bayesian Non-informative Prior } & \multicolumn{2}{|c|}{$\mathrm{SOE}^{\#}$} & \multicolumn{2}{|c|}{ MLR } \\
\hline & & ELISA & iPro Cube & ELISA & iPro Cube & ELISA & $\begin{array}{l}\text { iPro } \\
\text { Cube }\end{array}$ & ELISA & iPro Cube \\
\hline \multirow{6}{*}{$\begin{array}{l}\text { Salivary } \\
\text { cortisol } \\
\left(\mathrm{nMol} \cdot \mathrm{L}^{-1}\right)\end{array}$} & \multirow{2}{*}{ Pre-exercise } & $0.75(0.56,0.84)$ & $0.79(0.64,0.88)$ & $0.81(0.61,0.91)$ & $0.81(0.58,0.92)$ & $8.00 \%$ & $2.53 \%$ & $0.80(0.58,1.02)$ & $0.82(0.72,0.91)$ \\
\hline & & $0.53(0.21,0.72)$ & & $0.58(0.21,0.80)$ & & $9.43 \%$ & & $0.59(0.35,0.83)$ & \\
\hline & \multirow{2}{*}{$\begin{array}{c}\text { Post- } \\
\text { exercise }\end{array}$} & $0.54(0.23,0.71)$ & $0.75(0.60,0.85)$ & $0.59(0.25,0.80)$ & $0.77(0.51,0.90)$ & $9.26 \%$ & $2.67 \%$ & $0.59(0.30,0.87)$ & $0.77(0.62,0.92)$ \\
\hline & & $0.81(0.62,0.89)$ & & $0.87(0.69,0.94)$ & & $7.41 \%$ & & $0.87(0.77,0.97)$ & \\
\hline & \multirow{2}{*}{$\begin{array}{l}30 \text { min post- } \\
\text { exercise }\end{array}$} & $0.53(0.20,0.71)$ & $0.82(0.67,0.90)$ & $0.59(0.21,0.81)$ & $0.84(0.63,0.93)$ & $11.32 \%$ & $2.43 \%$ & $0.59(0.32,0.86)$ & $0.85(0.72,0.98)$ \\
\hline & & $0.71(0.47,0.83)$ & & $0.78(0.53,0.90)$ & & $9.86 \%$ & & $0.78(0.64,0.92)$ & \\
\hline \multirow{6}{*}{$\begin{array}{l}\text { Salivary a- } \\
\text { amylase } \\
\left(\mathrm{nKat} \cdot \mathrm{L}^{-1}\right)\end{array}$} & \multirow{2}{*}{ Pre-exercise } & $0.44(0.15,0.65)$ & $0.51(0.37,0.64)$ & $0.47(0.10,0.73)$ & $0.23(-0.23,0.60)$ & $6.81 \%$ & $54.90 \%$ & $0.47(0.16,0.77)$ & $0.23(-0.37,0.82)$ \\
\hline & & $0.32(0.10,0.56)$ & & $0.29(-0.12,0.62)$ & & $9.38 \%$ & & $0.29(0.07,0.52)$ & \\
\hline & \multirow{2}{*}{$\begin{array}{l}\text { Post- } \\
\text { exercise }\end{array}$} & $0.46(0.16,0.66)$ & $0.50(0.36,0.63)$ & $0.50(0.13,0.74)$ & $0.24(-0.19,0.37)$ & $8.69 \%$ & $52.00 \%$ & $0.49(0.26,0.73)$ & $0.24(-0.19,0.68)$ \\
\hline & & $0.37(0.11,0.62)$ & & $0.38(-0.14,0.72)$ & & $2.70 \%$ & & $0.39(-0.04,0.82)$ & \\
\hline & \multirow{2}{*}{$\begin{array}{l}30 \text { min post- } \\
\text { exercise }\end{array}$} & $0.33(0.10,0.57)$ & $0.77(0.62,0.86)$ & $0.31(-0.09,0.62)$ & $0.80(0.55,0.92)$ & $6.06 \%$ & $3.90 \%$ & $0.31(.003,0.61)$ & $0.81(0.57,1.05)$ \\
\hline & & $0.25(0.09,0.51)$ & & $0.13(-0.33,0.53)$ & & $48.00 \%$ & & $0.13(-0.15,0.40)$ & \\
\hline
\end{tabular}

2

3 Note: the two correlation coefficients presented for each variable in the ELISA column are those between the ELISA and each of the two iPro

4 Cube samples. The single value for each variable in the iPro Cube column is the correlation coefficient for the two iPro Cube samples; $95 \%$

5 credibility intervals are presented in parentheses; MLR = robust maximum likelihood estimator; ELISA = analysis via ELISA immunoassay; iPro

6 Cube $=$ correlation between duplicate samples via the point of care assessment; ${ }^{\#}$ SOE $($ size of effect $)=[$ initial prior $/$ default $/$ non-informative

7 prior)/ initial prior] * 100 (see Depaoli \& van de Schoot, 2017); 